\title{
Network Efficiency - Optimized Automaton Approach
}

\author{
K. Thiagarajan, \\ Department of Mathematics, \\ PSNA College of Engineering and \\ Technology, \\ Dindigul, India
}

\author{
N. Subashini, \\ Department of Mathematics, \\ SRM University, \\ Chennai, India
}

\author{
M. S. Muthuraman, \\ Department of Mathematics, \\ PSNA College of Engineering and \\ Technology, \\ Dindigul, India
}

\begin{abstract}
A sperner's grid is thought of a finite state system, where in the model gives rise to an optimal network through characterization of paths .the automation graphs of the various states gives rise to different groomable light paths in network.
\end{abstract}

Keywords-Automaton; Network; Efficiency; Characterization

\section{INTRODUCTION}

In recent years with the use of different kinds of communication, the network has gained popularity. Automaton traffic engineering is an effective solution to control network conjestion. Automaton traffic engineering comprises of scientific principles thus providing optimal characterization in network.

A network is represented by a set of nodes, inks between the nodes interconnecting them Destination nodes refer to traffic entering or leaving a node, transit nodes refer to nodes were no traffic can enter or leave the node.

\section{A. Network via Automata}

A finite state system represents a mathematical model of a system with certain input. The model finally gives a certain output. The input given to the machine is processed by various states; these states are called intermediate states. This intermediate state leads to final state.

A finite state Automata or simply a network consists of directed graphs composed of states and arcs. We start with a single initial state and could have any number of final states. A path is observed as a sequence of arcs or links from start state to final state.

\section{B. Network through Directions}

Consider a directed graph as $\mathrm{D}=$ (V (D), $\mathrm{X}$ $\left.(D), \chi_{D}\right)$, Consisting of nonempty set V (D), X (D) being disjoint from $V(D)$ and $\chi_{D}$ a function which associates $V(D)$ and $X(D)$. The set $V(D)$ is called the vertex set of $D$ and $X$ (D) its link set Elements of V (D) are taken as nodes of D and elements of X (D) are called links of (D).

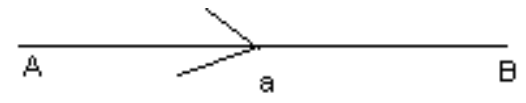

$a=(A, B)$ is a link of directed graph $D$ then ' $a$ ' is said to join $\mathrm{A}$ and $\mathrm{B}$. We consider only links through directions.

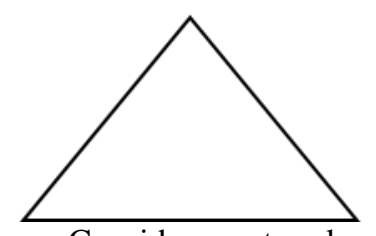

Consider a network as a triangular grid having nodes 0,1 , 2. Nodes could be taken within this grid. We consider only one node taken inside this grid. This single node can be either of 0,1 or 2 . In this paper we consider a single node within the grid. Considering a node within the grid, then the edged would be in order $0-1,1-2,2-0$. We consider ' 0 ' as a single node taken inside the grid, the direction 0 to 1 is taken as traffic in and 1 to o as out traffic. Similar aspects could be thought with regards to the other two namely 1 and 2. Edges with similar nodes are taken as ignored links.

If ' $n$ ' is the number of nodes taken on the side of triangular grid having end nodes 0 and 1 , the number networks having 010 is $2 n+1$, the number of nodes having nodes 01010 is ' $n$ ' and the number having nodes 0101010 is (n-1).

The maximum of 3 would exits as combination of 01 , as we consider a triangular grid. Considering 010, 01010, 0101010 we would have a graph of $\mathrm{P}_{2}, \mathrm{P}_{4}$, and $\mathrm{P}_{6}$ where $\mathrm{P}_{\mathrm{K}}$ refers to directions in the graph $\mathrm{K}$ being even. Finite Automaton is obtained for each of the graphs of 010, 01010, and 0101010 . The finite automaton associated with a directed graph is called a transition graph. The vertices of the graph correspond to the states.

\section{Nomenclature}

$\mathrm{q}_{\mathrm{i}-\text { State }}$

$\alpha$ - Number of directions towards the starting node of a path

C-Total number of networks

S- Number of directions towards the ending. Node of a path $\bar{p}$ - Efficiency

\section{Note}

1) Efficiency the ratio of the number of directions, towards the starting node with respect to the different between the total number of the networks of networks and number of directions, towards the ending node of the path .

2) Self loops are considered as ignored only in graphical representation self looping is considered in state diagrams. 


\section{GENERATION AND EFFICIENCY OF 010 NETWORK}

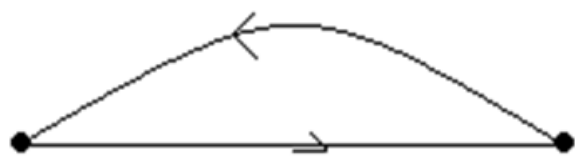

0

\section{1}

The automation for the above graph has the starts $\mathrm{q}_{0}, \mathrm{q}_{1}$ as described below

\section{A. State- $q_{0}$}

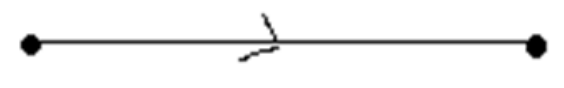

0

1

$\mathrm{q}_{0}$ is the initial state infers the path 0 to $1 \&$ is in itself under 01

B. State $-q_{1}$

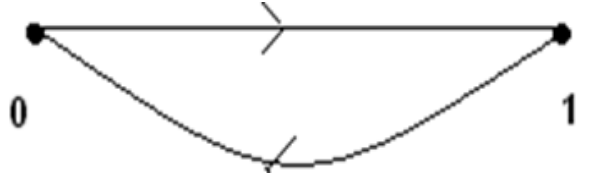

01

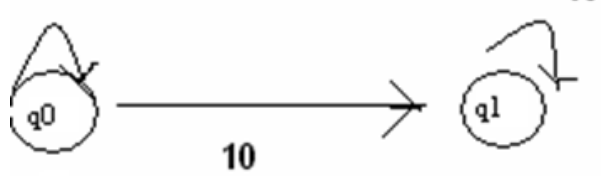

$\mathrm{q}_{1}$ is the state inferring 1 to 0 under the state $\mathrm{q}_{0}$. Thus $\mathrm{q}_{0}$ goes to $\mathrm{q}_{1}$ under 10

$$
{ }_{\mathrm{q}_{0}} \begin{array}{cc}
01 & 10 \\
\mathrm{q}_{1} & \left(\begin{array}{cc}
\mathrm{q}_{0} & \mathrm{q}_{1} \\
-- & \mathrm{q}_{1}
\end{array}\right)
\end{array}
$$

$\mathrm{q}_{0}$ goes to itself under $01 \& \mathrm{q}_{1}$ is in itself under 10

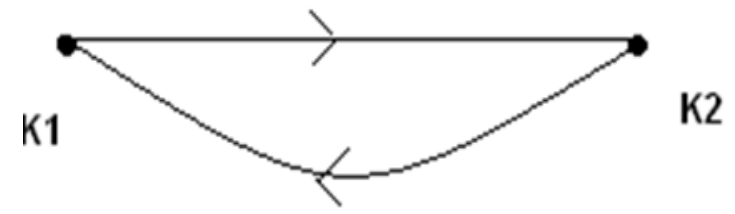

$\mathrm{K} 1$ represents the node $1 \& \mathrm{~K} 2$ represents the node 0

C. Characterization of the path $K 2$ to $K 1$

$\mathrm{C}=2$

$\alpha \rightarrow \mathrm{K} 2=1$

$\mathrm{S} \rightarrow \mathrm{K} 1=1$

$\bar{p}=1$

\section{GENERATION AND EFFICIENCY OF 01010}

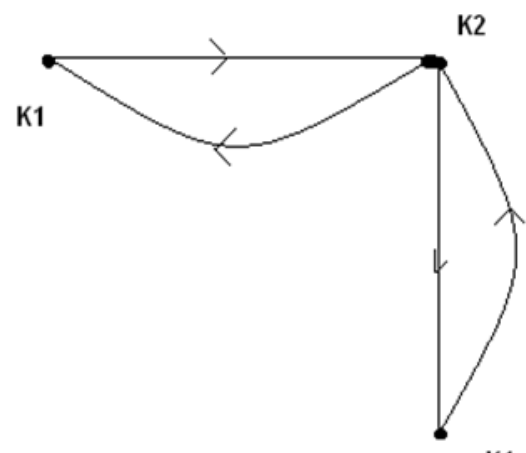

K1

$\mathrm{K} 1$ represents 1; K2 represents $0 ; \mathrm{K} 3$ represents 1 the above graph represents 0 to $1 ; 1$ to $0 \& 0$ to $1 \& 1$ to 0 thus giving the networks 01010. The automation for the above graph has the states $\mathrm{q}_{0}, \mathrm{q}_{1}, \mathrm{q}_{2}$, and $\mathrm{q}_{3}$.

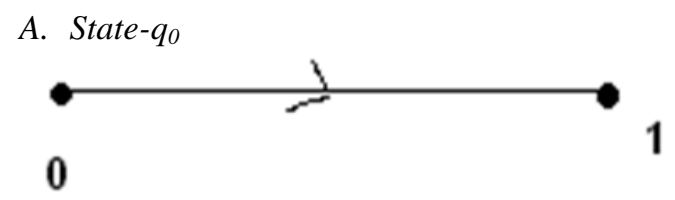

$\mathrm{q}_{0}$ is the initial state infers the path 0 to 1 and is in itself under 01 .

B. State- $q_{1}$

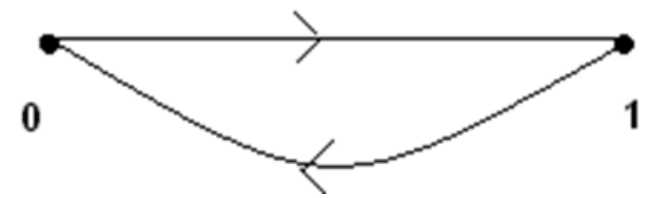

$\mathrm{q}_{1}$ is the state inferring 1 to 0 under the state $\mathrm{q}_{0}$. Thus $\mathrm{q}_{0}$ goes to $\mathrm{q}_{1}$ under $10, \mathrm{q}_{1}$ remains in itself under 10 .

C. State $-q_{2}$

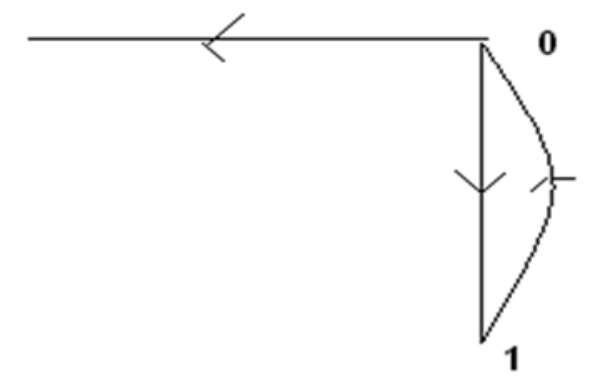

$\mathrm{q}_{2}$ is a state inferring 0 to 1 under the state $\mathrm{q}_{1}$, thus $\mathrm{q}_{1}$ goes to $\mathrm{q}_{2}$ under $01, \mathrm{q}_{2}$ remains in itself under 10 . 
D. State- $q_{3}$

1

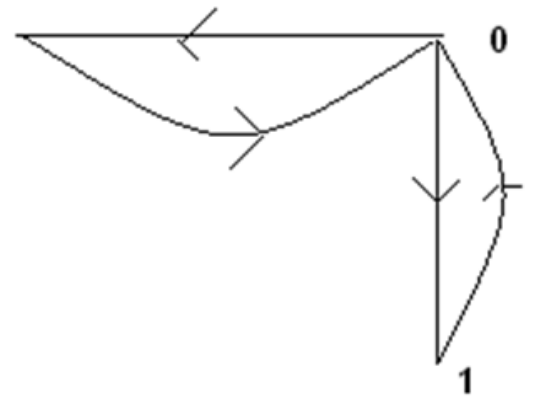

$\mathrm{q}_{3}$ is the state inferring 1 to 0 under the state $\mathrm{q}_{2}$, Thus $\mathrm{q}_{2}$ goes to $\mathrm{q}_{3}$ under $10, \mathrm{q}_{3}$ remains in itself under 10 .

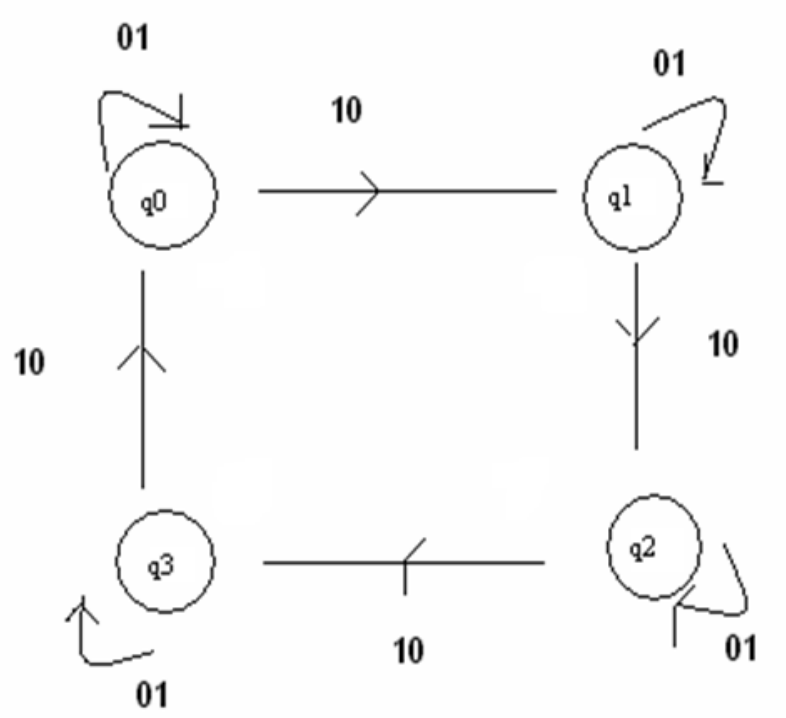

The matrix of the states with regards to the path $01 \& 10$

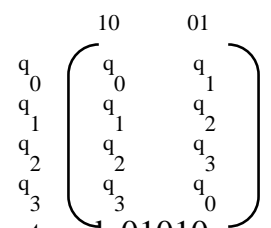

Efficiency of the network 01010

$$
\mathrm{C}=4
$$

E. Characterization of the path $K 1$ to $K 2$

$\mathrm{C}=4$

$\alpha \rightarrow \mathrm{K} 1=2$

$\mathrm{S} \rightarrow \mathrm{K} 2=2$

$\bar{p}=1$

F. Characterization of the path $\mathrm{K} 2$ to $\mathrm{K} 3$

$\mathrm{C}=4$

$\alpha \rightarrow \mathrm{K} 2=2$

$\mathrm{S} \rightarrow \mathrm{K} 3=2$

$\bar{p}=1$
G. Characterization of the path K2to K1

$\mathrm{C}=4$

$\alpha \rightarrow \mathrm{K} 2=2$

$\mathrm{S} \rightarrow \mathrm{K} 1=2$

$\bar{p}=1$

$H$. Characterization of the path K3to K1

$\mathrm{C}=4$

$\alpha \rightarrow \mathrm{K} 3=2$

$\mathrm{S} \rightarrow \mathrm{K} 1=2$

$\bar{p}=1$

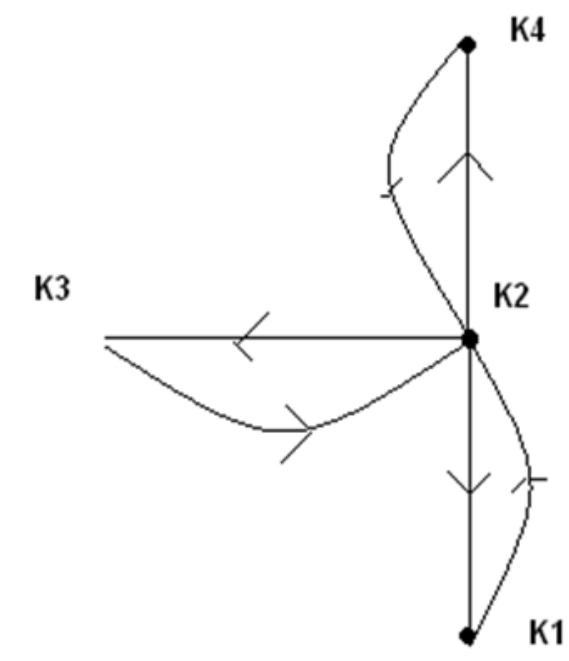

IV. GENERATION AND EFFICIENCY OF 0101010 NETWORK

K1 Represents 1; K2 represents 0; K3 Represents 1; K4 Represents 1.The automation for the above graph has the states $\mathrm{q}_{0}, \mathrm{q}_{1}, \mathrm{q}_{2}, \mathrm{q}_{3}$, and $\mathrm{q}_{4}$.

A. State- $q_{0}$

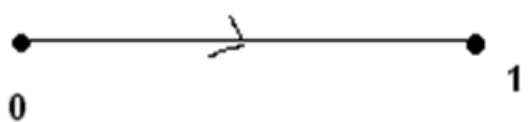
01.

$\mathrm{q}_{0}$ is the initial state infers the path 0 to $\mathrm{q}_{0}$ is in itself under

B. State- $q_{1}$

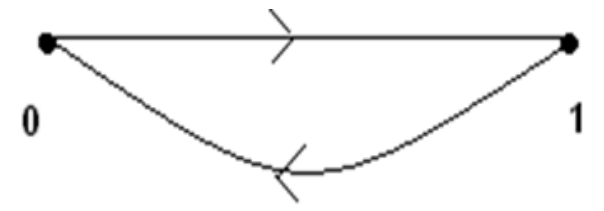

$\mathrm{q}_{1}$ is the state inferring 1 to 0 under the state $\mathrm{q}_{0}$. Thus $\mathrm{q}_{0}$ goes to $q_{1}$ under $10, q_{1}$ remains in itself under 10 . 
C. State- $q_{2}$

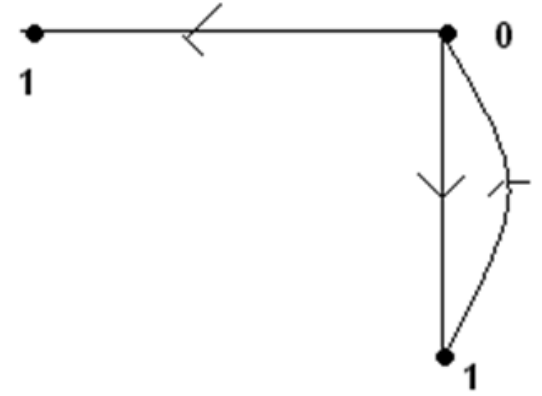

$\mathrm{q}_{2}$ is a state inferring 0 to 1 under the state $\mathrm{q}_{1}$, thus $\mathrm{q}_{1}$ goes to $\mathrm{q}_{2}$ under $01, \mathrm{q}_{2}$ remains in itself under 10 .

D. State $-q_{3}$

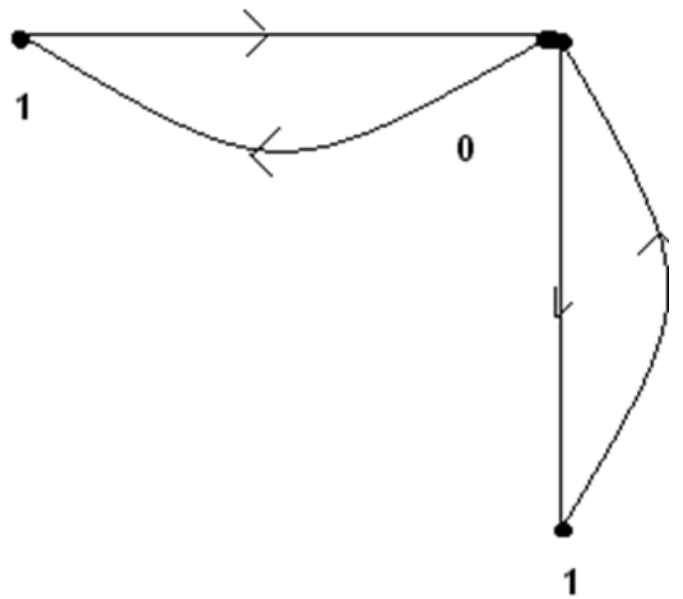

$\mathrm{q}_{3}$ is the state inferring 1 to 0 under the state $\mathrm{q}_{2}$, Thus $\mathrm{q}_{2}$ goes to $\mathrm{q}_{3}$ under $10, \mathrm{q}_{3}$ remains in itself under 10 .

E. State- $q_{4}$

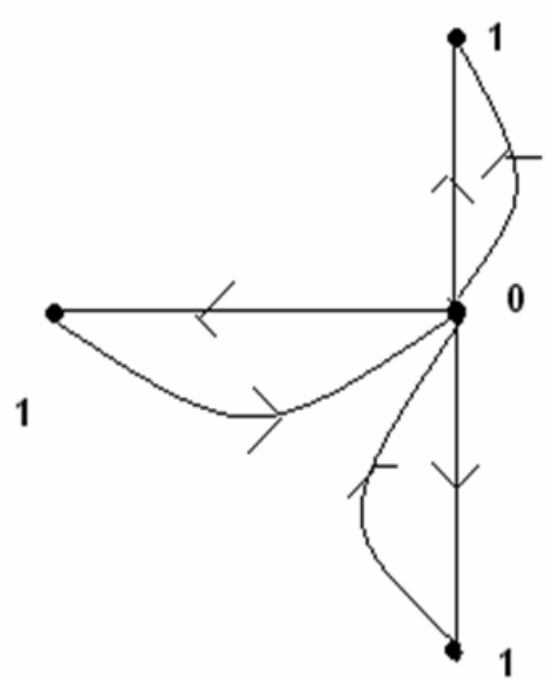

$\mathrm{q}_{4}$ is a state inferring 0 to 1 under the state $\mathrm{q}_{3}$, thus $\mathrm{q}_{3}$ goes to $\mathrm{q}_{4}$ under $01, \mathrm{q}_{4}$ remains in itself under 10 .
F. State- $q_{5}$

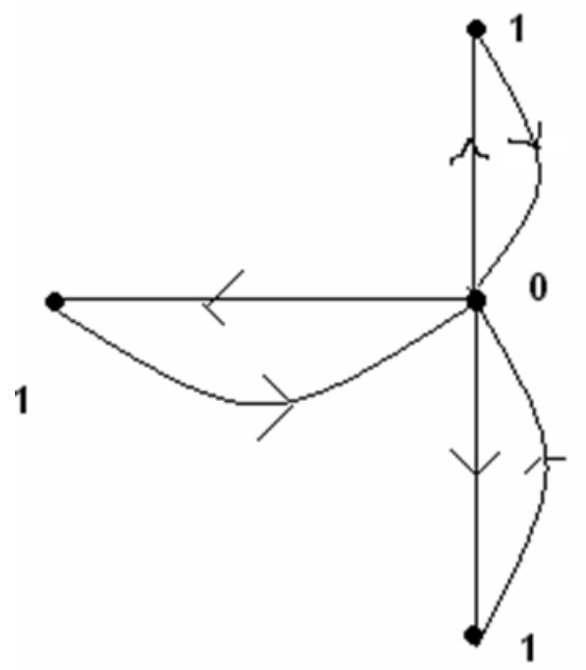

$\mathrm{q}_{5}$ is a state inferring 0 to 1 under the state $\mathrm{q}_{4}$, thus $\mathrm{q}_{4}$ goes to $\mathrm{q}_{5}$ under $01 . \mathrm{q}_{5}$ remains in itself under 10 .

The matrixes of the states are as fallows

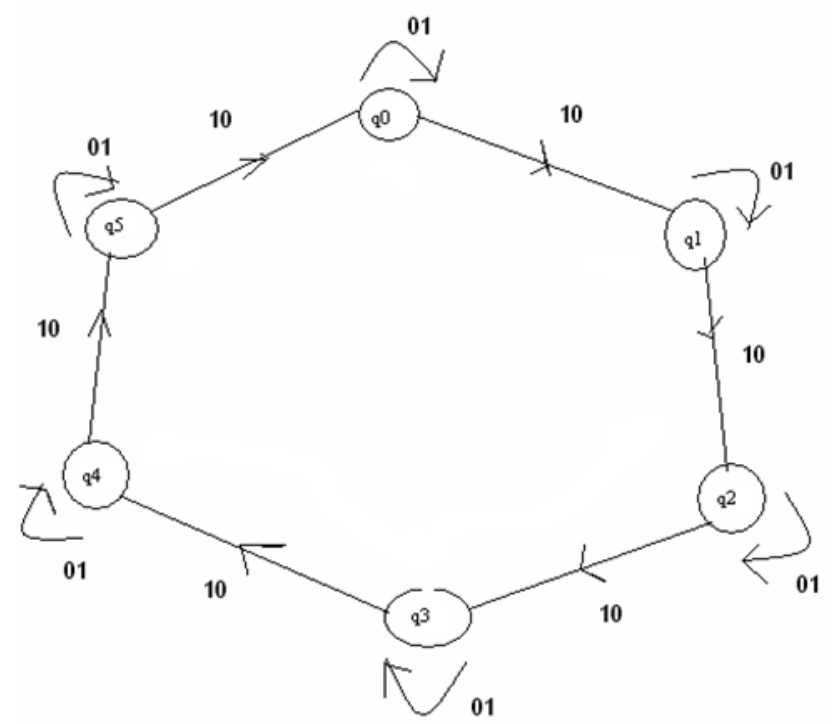

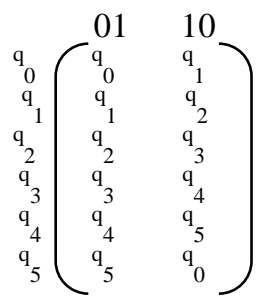

Efficiency of the network 0101010

\section{G. Characterization of $K 1$ to $K 2$}

$\mathrm{K} 2$ to $\mathrm{K} 1$

$\mathrm{K} 3$ to $\mathrm{K} 2$

$\mathrm{K} 2$ to $\mathrm{K} 3$

$\mathrm{K} 2$ to $\mathrm{K} 4$

$\mathrm{K} 4$ to $\mathrm{K} 2$ 
$\mathrm{C}=6$

$\alpha \rightarrow \mathrm{K} 1=3$

$\alpha \rightarrow \mathrm{K} 2=3$

$\alpha \rightarrow \mathrm{K} 3=3$

$\alpha \rightarrow \mathrm{K} 4=3$

$\mathrm{S} \rightarrow \mathrm{K} 1=3$

$\mathrm{S} \rightarrow \mathrm{K} 2=3$

$\mathrm{S} \rightarrow \mathrm{K} 3=3$

$\mathrm{S} \rightarrow \mathrm{K} 4=3$

\section{H. Characterization of $K 1$ to $K 2$}

\section{$\mathrm{K} 2$ to $\mathrm{K} 1$}

$\mathrm{K} 3$ to $\mathrm{K} 2$

$\mathrm{K} 2$ to $\mathrm{K} 3$

$\mathrm{K} 2$ to $\mathrm{K} 4$

$\mathrm{K} 4$ to $\mathrm{K} 2$

In the of the above case we obtain $\bar{p}=1$

I. Result

We observe from the automation of the graphs 010; 01010; 0101010 the state $\mathrm{q}_{\mathrm{i}} ; \mathrm{i}=1,2,3,4$ goes to $\mathrm{q}_{\mathrm{i}}+1$

In the Graphical representation we observe

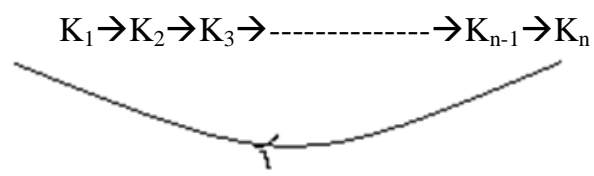

In general $\mathrm{K}_{\mathrm{i}} \rightarrow \mathrm{K}_{\mathrm{i}+1}$ under the path 01

\section{CONCLUSION}

We observe that in the graphical representation of the graphs 010; 01010; 0101010; we observe in the characterization of the path $\mathrm{K}_{1}$ to $\mathrm{K}_{2} \& \mathrm{~K}_{2}$ to $\mathrm{K}_{1}$ efficiency is 1.In the graphical representation of $01010 \& 0101010$ we observe the characterization of the path $\mathrm{K}_{2}$ to $\mathrm{K}_{3} \& \mathrm{~K}_{3}$ to $\mathrm{K}_{2}$ the efficiency is 1.Both the networks are of the source and destination groom able light paths, Wherein the termination is at the node $0 \&$ could be routed towards other nodes, \& can also originate from other nodes. When we consider efficiency we observe that destination is at the node $0 \& 1$ according to the consideration of the path automata is applied to paths of a network there by giving ideas for better groom ability

\section{ACKNOWLEDGMENT}

The authors would like to thank Dr. Ponnammal Natarajan, Former Director - Research, Anna University- Chennai, India and currently an Advisor, (Research and Development), Rajalakshmi Engineering College, Dr. K. Sarukesi former vice chancellor in Hindustan University-Chennai, India, Professor E.Sampath Kumar Acharya and Professor L.Pushpalatha, University of Mysore, Mysore, for this initiative ideas and fruitful discussions with respect to the paper's contribution.

\section{REFERENCE}

[1] Graph Theory by Frank Harary, Addison Wesley Publishing Company, Third Printing, October (1972).

[2] Graph Theory With Applications To Engineering And Computer Science By Narsingh Deo, PHI Publications, New Delhi (1995).

[3] Special Study in (1,2 )- Distance Coloring and (1,2) -Distance Graphs. K.Thiagarajan, L.Pushpalatha, Ponnamal Natarajan. Ultra Scientist Of Physical Sciences, Vol.19 (3) M.731-733(2007).

[4] Special Study in (2, 2) Distance Coloring and (2,2) -Distance Graphs (for paths and cycles). K.Thiagarajan, Ponnamal Natarajan. Ultra Scientist Of Physical Sciences, Vol. 19 (3) M.734-736(2007).

[5] Introduction to Automata Theory, Languages, and Computation( $3^{\text {rd }}$ Edition Pearson Publication) -John E. Hopcroft, Rajeevmotwani, Jeffrey D. Ullman.

\section{Authors PROFILE}

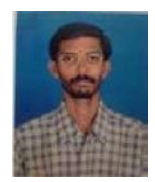

K. Thiagarajan completed his Ph.D from University of Mysore. He is currently working as Associate Professor in the Department of Mathematics, P.S.N.A.College of Engineering and Technology, Dindigul, Tamilnadu, India. $\mathrm{He}$ has totally 18 years of experience in teaching. He has attended and presented research articles in 37 National and International Conferences and published one National journal and more than 50 International journals, Currently he is working on Big Data and web mining through automata and set theory. His area of specialization is 'Coloring of Graphs and DNA Computing.

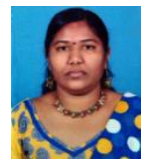

Ms. R. Subashini have completed M .sc, B Ed, M phil. and she is having 10 years teaching experience in various engineering colleges. Currently she is working as a Assistant Professor in Department of Mathematics at SRM University, Kattankulathur, Chennai. She have Participated and presented two papers in national and international conferences at SRM and Sathyabama university. Currently she is working in optimization of networks.

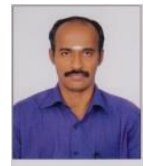

M.S.Muthuraman (B.Sc., M.Sc., M.Phil., in Mathematics)is currently working as an Associate Professor ,Department of Mathematics, PSNA College of Engineering and Technology,Dindigul, Tamilnadu, India. He is the author of 25 papers published in reputed journals and in proceedings of conferences, with many references from other researchers. His interests include Fuzzy Algebra, Discrete

Mathematics. 